\title{
Changing patterns in HIV-1 non-B clade prevalence and diversity in ltaly over three decades*
}

\author{
A Lai, ${ }^{1 \dagger} \mathrm{C}$ Riva, ${ }^{1 \dagger} \mathrm{A}$ Marconi, ${ }^{2} \mathrm{M}$ Balestrieri, ${ }^{2} \mathrm{~F}$ Razzolini, ${ }^{2} \mathrm{G}$ Meini, ${ }^{2} 1$ Vicenti, ${ }^{2} \mathrm{~A}$ Rosi, ${ }^{2} \mathrm{~F}$ Saladini, ${ }^{2} 1 \mathrm{Caramma},{ }^{1}$ \\ M Franzetti, ${ }^{1}$ V Rossini, ${ }^{1}$ A Galli, ${ }^{1}$ M Galli, ${ }^{1}$ M Violin, ${ }^{1}$ M Zazzi ${ }^{2}$ and C Balotta ${ }^{1}$ \\ ${ }^{1}$ Section of Infectious Diseases and Immunopathology, Department of Clinical Sciences, 'L. Sacco' Hospital, University \\ of Milan, Milan, Italy and ${ }^{2}$ Section of Microbiology, Department of Molecular Biology, University of Siena, Siena, Italy
}

\begin{abstract}
Background
HIV-1 non-B subtypes have recently entered Western Europe following immigration from other regions. The distribution of non-B clades and their association with demographic factors, over the entire course of the HIV-1 epidemic, have not been fully investigated in Italy.
\end{abstract}

\section{Methods}

We carried out a phylogenetic analysis of HIV-1 pol sequences derived from 3670 patients followed at 50 Italian clinical centres over nearly three decades.

\section{Results}

Overall, 417 patients (11.4\%) carried non-B subtypes. The prevalence of non-B strains increased from $2.6 \%$ in $1980-1992$ to $18.9 \%$ in $1993-2008(P<0.0001)$ in a subset of 2479 subjects with a known year of diagnosis. A multivariate analysis on a subset of 1364 patients for whom relevant demographic data were available indicated that African ethnicity, heterosexual route of infection and year of diagnosis were independently associated with non-B HIV-1 infection $(P \leq 0.0001)$. All pure subtypes, except for clade $\mathrm{K}$, and seven circulating recombinant forms were detected, accounting for 56.6 and $34.1 \%$ of the non-B infections, respectively. The F1 subtype was the most prevalent non-B clade among Europeans and was acquired heterosexually in half of this patient population. Unique recombinant forms accounted for $9.4 \%$ of the non-B sequences and showed a $\mathrm{B} / \mathrm{F} 1$ recombination pattern in one-third of cases.

\section{Conclusions}

The circulation of non-B clades has significantly increased in Italy in association with demographic changes. Spread of the F1 subtype and B/F recombinants appears to predominate, which may result in a redistribution of the relative proportions of the different strains, and this could lead to overlapping epidemics. Thus, the HIV-1 landscape in Italy may in future be distinct from that of the rest of Europe.

Keywords: HIV-1, new diagnoses of HIV-1 infection, recombinants, subtypes, variability

Accepted 19 January 2010

\section{Introduction}

Nine discrete lineages of group M HIV-1 (A-D, F-H, $\mathrm{J}$ and $\mathrm{K}$ ) have differentiated during the global pandemic as a result of massive virus replication, the very high error rate of reverse transcriptase (RT) and the selective pressure

*This work was presented in part at the 7th European HIV Drug Resistance Workshop held in Stockholm, Sweden, 25-27 March, 2009.

Correspondence: Dr Alessia Lai, Section of Infectious Diseases and Immunopathology, Department of Clinical Sciences, 'L. Sacco' Hospital, University of Milan, Via G.B. Grassi, 74, 20157 Milan, Italy. Tel: + 39 02503 19775; fax: + 3902503 19768; e-mail: alessia.lai@unimi.it

${ }^{\dagger}$ Contributed equally to this work. exerted by the immune system. The highly recombinogenic activity of HIV-1 RT has added further complexity to the global diversity of HIV-1 as 43 circulating recombinant forms (CRFs) have already been characterized and a number of unique recombinant forms (URFs) have been identified world-wide [1-3]. Most subtypes and CRFs were originally restricted to specific geographical regions or populations, but their distribution is constantly evolving [4]. In order to monitor the evolution of the global pandemic, it is convenient and effective to assign viral clades, which allow evaluation of the local epidemiological trends that result from social changes and migration flows. 
On the basis of available data, subtype B of HIV-1 entered first in Western Europe as well as in the United States, Canada and Australia and has been the dominant subtype for about two decades [5]. However, over the past few years, several studies have reported that non-B strains have entered and are circulating in several previously B-restricted areas [6-13]. The recent epidemiology of HIV-1 infection in Western European countries with large immigrant communities has been characterized by increasing genetic diversity and a marked rise in non-B subtype strains among newly diagnosed individuals [14-17]. It has been assumed that most non-B subtype infections in Western Europe are linked to migration from or travel to areas of high HIV-1 prevalence, mainly sub-Saharan Africa, South America and, to a lesser extent, South-East Asia, where non-B clades predominate. This spread of non-B clades into Italy occurred at a time when epidemiological factors such as the ethnicity, route of infection and gender of the Italian HIV-1-infected population underwent profound changes. Sexual transmission has become the most common route of HIV-1 acquisition, while new infections among injecting drug users (IDUs) have substantially declined. Sexual acquisition of HIV-1 has shown a greater increase in heterosexuals than in men who have sex with men (MSM). As a consequence, the ratio of male to female HIV-1 prevalence has decreased over time [18].

At present, no official estimate of the rate of onward transmission of non-B subtypes is available, but the limited data suggest the acquisition of infection from individuals of non-Caucasian ethnicity. Information on the origin of non-B infections is limited because supporting epidemiological data have frequently been lacking or not thoroughly investigated. Molecular epidemiology can indicate the origin of an infection, reveal outbreaks within population subgroups, and provide a means of monitoring the spread of infection within and among different exposure groups [19,20].

The aim of this study was to evaluate the prevalence and distribution of non-B subtypes in a large HIV-1-infected cohort in Italy with sequence data generated at one reference laboratory. We assessed the temporal trends in non-B subtype circulation and evaluated the associations between non-B infection and the main demographic variables from 1980 to 2008. Furthermore, we investigated trends in the spread of non-B clades in Italy in relation to ethnicity, route of infection and gender.

\section{Materials and methods}

\section{Patients}

Overall, 3670 HIV-1-positive individuals, who had been referred to 50 clinical centres in 13 Italian regions in the period 1980-2008, were included in the study. Patients received a genotypic resistance test at diagnosis or prior to the start of therapy or at treatment failure. All the tests were performed at the HIV Monitoring Service of the Department of Molecular Biology of the University of Siena, Siena, Italy. Patients were included in the Antiretroviral Resistance Cohort Analysis (www.hivarca.net) database and provided informed consent to have their anonymized data stored on a central server. For each patient included in the analysis, the earliest available HIV-1 genotype was evaluated.

The date of HIV-1 diagnosis, established as the furst positive HIV-1 antibody test, was known for 2479 subjects of the 1980-2008 period [the 'HIV diagnosis' (HD) subset]. Demographic data (gender, risk category, country of origin, date of diagnosis and age) were collected by physicians in interviews with the patients and recorded in the database together with virological, immunological, treatment and clinical information. Country of origin, risk factor, gender and date of diagnosis were concomitantly available for a total of 1364 subjects of the 1980-2006 period [the 'complete data' (CD) subset], on whom correlation analyses were performed.

\section{Subtype assignment}

Subtyping was based on a partial HIV-1 pol sequence of 987 nucleotides, encoding the whole protease and amino acids 1-230 of RT. This region was amplified by RT-polymerase chain reaction and sequenced using infrared-labelled primers as previously described [21,22]. Sequences were first analysed using the National Center for Biotechnology Information HIV-1 subtyping tool to quickly discriminate between $B$ and non-B strains.

Non-B sequences were subsequently aligned with sequences from the most recent reference data set from the Los Alamos National Laboratory website (http://hiv. lanl.gov/) using BroEDIT 7.0.5 (www.mbio.ncsu.edu/BioEdit/ bioedit.html) and ClustalX 1.83 (http://bips.u-strasbg.fr/fr/ Documentation/ClustalX/). The resulting alignment was analysed with the PHYLIP package version 3.67 (http:// evolution.genetics.washington.edu/phylip.html) and a neighbour-joining tree was built based on the F84 substitution model. The reliability of the tree topology was assessed by bootstrapping using 1000 replicate data sets.

Sequences that could not be unequivocally assigned to a pure subtype or CRF were considered as possible recombinants and examined using SiMPLOT 3.5.1 (http://sray.med.som.jhmi. edu/SCRoftware/simplot/) to identify the recombination pattern. Similarity plots and bootscans were generated by comparing the sequence under investigation with those of reference strains using a sliding window of 300 nucleotides with 20-nucleotide steps. Subtype assignment of each recombination fragment was confirmed through phylogenetic analysis using the same parameters as for the whole sequences. 
For URFs, we further examined the degree of similarity of the pol sequences to other HIV-1 sequences, using the BLAST search engine (www.ncbi.nlm.nih.gov/blast/) with default settings.

\section{Statistical analysis}

Standard nonparametric methods (the Wilcoxon signedrank test) were used to compare median age, HIV-1 RNA levels and CD4 cell counts in patients with B and non-B subtypes. Categorical variables in these two groups were compared using $\chi^{2}$ or Fisher's exact test. The crude and Mantel-Haenszel adjusted odds ratios of having a non-B subtype were also calculated. Univariate analysis was performed using $\chi^{2}$ and logistic regression. A subsequent multivariate analysis was performed on all variables, using the same tests with a full model. The Cochran-Armitage test for trend was used to determine if an association, when present, was linear. In all tests, a $P$-value below 0.05 was considered significant.

\section{Results}

\section{Characteristics of patient subsets}

HIV-1 subtype was determined for all patients, revealing an overall prevalence of non-B clades of 11.4\% (417 of 3670 patients). Continent of origin (92.2\% Europe, 4.5\% Africa and $3.3 \%$ other), route of infection (35.6\% heterosexual, 32.9\% IDU, 26.3\% MSM and 5.2\% other) and gender (70.4\% male) were known for 97\% $(n=3561), 53.5 \%(n=1963)$ and $98.1 \%$ ( $n=3602)$ of individuals, respectively. Overall, the patients had a median age of 38 years [interquartile range (IQR) 33-44 years]. The median CD4 cell count and HIV-1 plasma viral load at genotype testing were 305 cells/ $\mu \mathrm{L}$ (IQR 150-487 cells/ $\mu \mathrm{L}$ ) and 4.15 log HIV-1 RNA copies/mL (IQR 3.23-4.89 log copies $/ \mathrm{mL}$ ), respectively.

Figures for patients in the HD subset were similar. Ethnicity, route of infection and gender were known for $99.1 \%(n=2457), 55.1 \%(n=1365)$ and $99.2 \%(n=2461)$ of individuals, respectively. The continent of origin was mainly Europe (92.3\%), with Africa accounting for 4.6\% and other continents for $3.1 \%$ of patients. Risk factor for HIV infection was IDU for 35.7\% of patients, heterosexual for 33.8\%, and MSM for 24.4\%. In this group, $69.3 \%$ of patients were male. The median age (37 years; IQR 33-43 years), CD4 cell count (306 cells/ $\mu \mathrm{L}$; IQR 142-488 cells/ $\mu \mathrm{L}$ ) and viral load (4.11 log copies/mL; IQR 3.2-4.9 log copies/mL) were also not different from those of the whole patient population.

Demographics and laboratory data of the CD subset, stratified according to viral subtype, are shown in Table 1. All the patient characteristics considered were similarly
Table 1 Demographic, virological and immunological characteristics of the 1364 patients with complete data available (subset CD)

\begin{tabular}{|c|c|c|c|c|}
\hline & $\begin{array}{l}\text { All } \\
\text { patients } \\
(n=1364)\end{array}$ & $\begin{array}{l}\text { B subtype } \\
(n=1241)\end{array}$ & $\begin{array}{l}\text { Non-B } \\
\text { subtypes } \\
(n=123)\end{array}$ & $P$-value \\
\hline Ethnic group [\% $(n)]$ & & & & $<0.0001^{*}$ \\
\hline European & 93.5 (1275) & $97.8(1214)$ & $49.6(61)$ & \\
\hline African & $4.3(58)$ & $0.2(3)$ & $44.7(55)$ & \\
\hline Other ${ }^{\dagger}$ & $2.3(31)$ & $2.0(24)$ & $5.7(7)$ & \\
\hline Risk factor $[\%(n)]$ & & & & $<0.0001^{*}$ \\
\hline Injecting drug use & $35.8(488)$ & $38.9(483)$ & $4.1(5)$ & \\
\hline Heterosexual sex & $33.7(460)$ & $29.4(365)$ & 77.2 (95) & \\
\hline $\begin{array}{l}\text { Men having sex } \\
\text { with men }\end{array}$ & $24.4(333)$ & $25.8(320)$ & $10.6(13)$ & \\
\hline Other ${ }^{\ddagger}$ & $6.1(83)$ & $5.8(73)$ & $8.1(10)$ & \\
\hline Gender $[\%(n)]$ & & & & $<0.0001^{*}$ \\
\hline Male & 69.5 (948) & $71.2(884)$ & $52.0(64)$ & \\
\hline Female & $30.5(416)$ & $28.8(357)$ & $48.0(59)$ & \\
\hline $\begin{array}{l}\text { Year of diagnosis } \\
{[\%(n)]}\end{array}$ & & & & $<0.0001^{*}$ \\
\hline 1980-1992 & $56.2(767)$ & $60.3(748)$ & $15.5(19)$ & \\
\hline $1993-2006$ & $43.8(597)$ & 39.7 (493) & $84.5(104)$ & \\
\hline $\begin{array}{l}\text { Age (years) } \\
\text { [median (IQR)] }\end{array}$ & $37(32-42)$ & $37(33-42)$ & $33(27-40)$ & $<0.0001^{\S}$ \\
\hline $\begin{array}{l}\text { HIV-1 RNA } \\
\text { (log copies/mL) } \\
\text { [median (IOR)] }\end{array}$ & $4.1(3.2-4.8)$ & $4.0(3.2-4.8)$ & $4.2(3.2-4.9)$ & $N S^{\S}$ \\
\hline 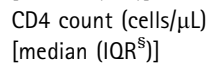 & $310(130-491)$ & ) 310 (129-486) & 324 (197-581) & $N S^{\S}$ \\
\hline $\begin{array}{l}\text { Percentage of } \\
\text { patients }\end{array}$ & 100.0 & 91.0 & 9.0 & - \\
\hline
\end{tabular}

${ }^{*} \chi^{2}$ test.

Other: Latin Americans and Asians.

${ }^{\ddagger}$ Other: transfusion, vertical transmission and unknown risk.

sWilcoxon test.

$I Q R$, interquartile range; NS, not significant.

distributed in the global population and in the HD and CD subsets. For these individuals the year of HIV-1 diagnosis covered the period 1980-2006. One hundred and twentythree of these individuals (9.0\%) harboured non-B subtypes.

Temporal trend for non-B subtypes in the period 1980-2008

The prevalence of infection with HIV-1 B and non-B clades over time was evaluated in patients of subset $\mathrm{HD}$, who were diagnosed in the period 1980-2008 (Fig. 1). Two hundred and fifty-seven (10.4\%) individuals harboured a non-B subtype. The test for trend indicated a significant association between infection with non-B strains and the year of diagnosis $(P<0.0001)$. This association was linear with an increasing trend. A regression analysis, modelling the probability of acquiring a non-B strain by calendar year, supported this trend and indicated that the odds of acquiring a non-B subtype were 1.27-fold higher per subsequent year (95\% confidence interval 1.23-1.31). 


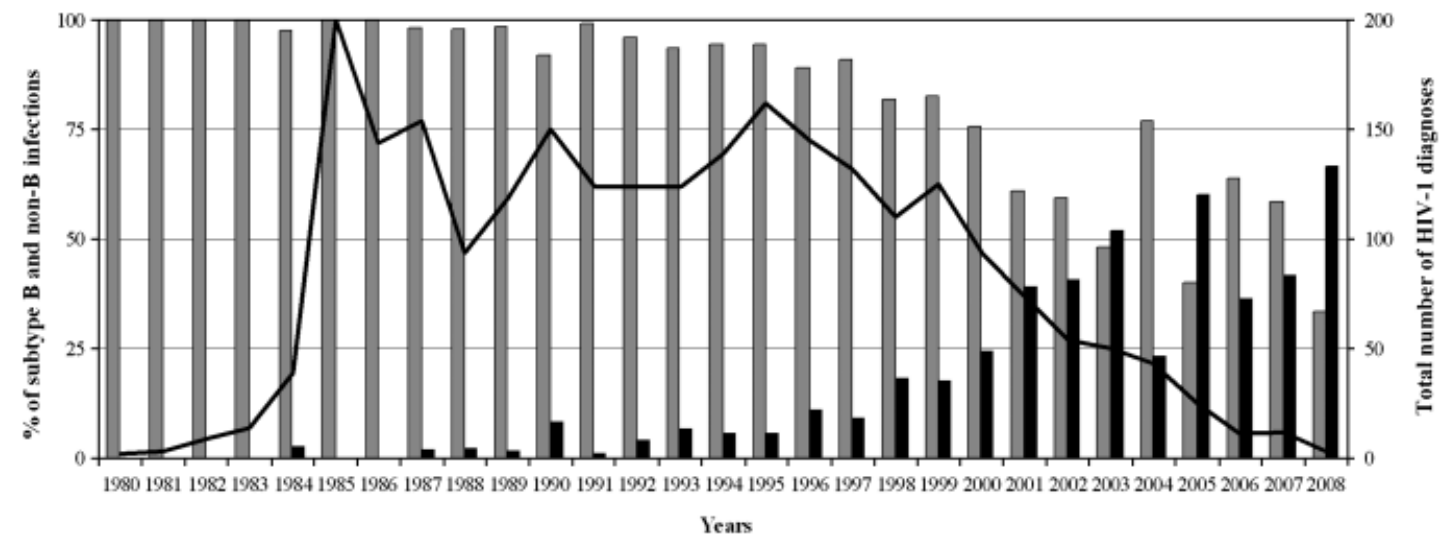

Fig. 1 Distribution of B and non-B HIV-1 infections over time in patients in the 'HIV diagnosis' subset. Black column, non-B infections; grey column, B infections. The continuous line indicates absolute numbers of infections diagnosed every year.

The first cases of infection with pure non-B subtypes, CRFs or URFs were detected in African individuals in 1984, 1990 and 1994, respectively. These patients, who migrated to Italy from Senegal, Burkina Faso and Ivory Coast, carried an A1 subtype, a CRF09_cpx strain and a CRF02_AG/A1 recombinant, respectively. The first European patients harbouring a pure non-B strain (A1), a CRF (01_AE) and a recombinant form (B/F) were diagnosed in 1987, 1996 and 1995, respectively.

Overall, 52.4\% of new HIV-1 diagnoses occurred before 1993. Thereafter, the number of new diagnoses has markedly decreased. Non-B strains were carried by only $2.6 \%$ (34 of 1300) of newly diagnosed patients before 1993 but by $18.9 \%$ (223 of 1179) in the period 1993-2008 $(P<0.0001)$.

Distribution of HIV-1 clades with respect to demographic variables over time

The demographics of two groups of patients in subset CD, those diagnosed before 1993 and from 1993 onwards, were then compared. In this subset, non-B subtypes accounted for 2.5\% (19 of 767) of HIV-1 diagnoses in 1980-1992 and for 17.4\% (104 of 597) in 1993-2006 $(P<0.0001)$. Although the proportions of patients of African, Latin American and South-East Asian origin significantly increased from 1.7\% $(n=14)$ in the period before 1993 to $12.6 \%(n=75)$ in the period from 1993 onwards $(P<0.0001)$, non-B subtypes markedly increased among Europeans from 1.9\% (13 of 753) in the earlier period to $9.2 \%$ (48 of 522) in the later period $(P<0.0001)$ (Fig. 2a).

Overall, the proportions of heterosexuals and MSM increased from $23.5 \%(n=180)$ in the earlier period to $46.9 \%(n=280)$ in the later period $(P<0.0001)$ and from $17.3 \%(n=133)$ to $33.5 \%(n=200)(P<0.0001)$, respectively, while the proportion of IDUs decreased from 52.9\% $(n=406)$ to $13.7 \%(n=82)(P<0.0001)$. The proportion of heterosexuals carrying a non-B variant increased from $7.8 \%$ (14 of 180 ) to $28.9 \%$ ( 81 of 280$)(P<0.0001)$ between the two study periods. An increase in the prevalence of non-B subtypes from $0.2 \%$ (one of 406) to $4.9 \%$ (four of 82$)(P=0.003)$ and from $0.8 \%$ (one of 133) to $6.0 \%$ (12 of 200) $(P=0.018)$ was observed in IDUs and MSM, respectively (Fig. 2b).

The gender distribution did not differ between the two periods [30.7\% $(n=236)$ and 30.2\% $(n=180)$ female, respectively]. A disproportionately high number of female patients was recorded among IDUs in both periods (data not shown). Nevertheless, female patients carrying non-B variants increased from 1.3\% (three of 236) in the period up to 1993 to 31.1\% (56 of 180) in the period from 1993 onwards $(P<0.0001)$ (Fig. $2 \mathrm{c})$.

\section{Factors associated with non-B infection}

The probability of acquiring a non-B subtype was also studied in patients with complete demographic data (subset CD) (Table 2). In the univariate analysis, a strong association was found between African origin and non-B clades (94.8\% of African people carried a non-B strain) $(P<0.0001)$, even though Europeans accounted for $49.6 \%$ of non-B-infected patients. The most prevalent risk category in subset CD was IDU (35.8\%), followed by heterosexual (33.7\%) and MSM (24.4\%). Nonetheless, a highly disproportionate percentage of non-B-infected patients were heterosexual $(77.2 \% ; P<0.0001)$. In the $\mathrm{CD}$ subset, $69.5 \%$ of patients were male. The gender distribution differed between groups infected with non-B and $B$ subtypes; patients harbouring a non-B strain showed a 1:1.1 male to female ratio compared with 2.5:1 for subtype B-infected individuals. Female gender was significantly associated with infection with a non-B strain $(P<0.0001)$, 

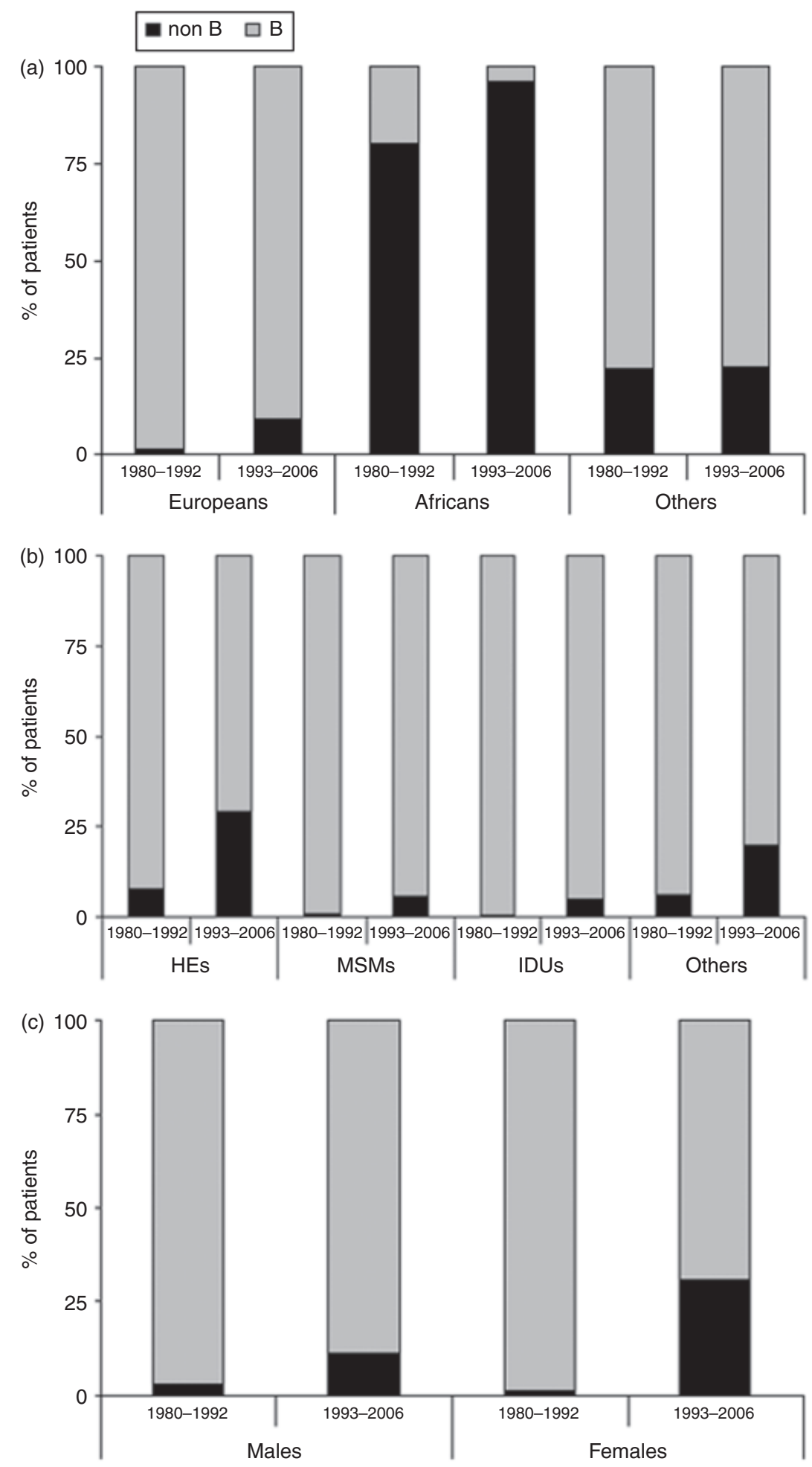

Fig. 2 Characteristics of patients infected with HIV-1 B and non-B subtypes. (a) Ethnicity, (b) route of infection and (c) gender for patients with non-B and B clades in the 1980-1992 and 1993-2006 periods are shown. HE, heterosexuals; IDU, injecting drug use; MSM, men who have sex with men. 
Table 2 Odds ratios (ORs) of harbouring a non-B HIV-1 clade in the 'complete data' (CD) patient subset ( $n=1364$ )

\begin{tabular}{|c|c|c|c|c|c|c|}
\hline \multirow[b]{2}{*}{ Covariate } & \multicolumn{3}{|c|}{ Univariable analysis } & \multicolumn{3}{|c|}{ Multivariable analysis } \\
\hline & $\mathrm{OR}$ & $95 \% \mathrm{Cl}$ & $P$ & OR & $95 \% \mathrm{Cl}$ & $P$ \\
\hline \multicolumn{7}{|l|}{ Ethnicity } \\
\hline European & - & - & - & - & - & - \\
\hline African & 364.9 & $111.0->999.9$ & $<0.0001$ & 149.9 & $42.5-528.6$ & $<0.0001$ \\
\hline Latin American & 3.5 & $1.0-12.3$ & 0.0275 & 3.8 & $1.0-14.6$ & 0.1646 \\
\hline Other & 11.4 & $3.2-39.9$ & 0.9482 & 7.9 & $1.9-32.0$ & 0.9504 \\
\hline \multicolumn{7}{|l|}{ Route of infection } \\
\hline Injecting drug use & - & - & - & - & - & - \\
\hline Heterosexual sex & 25.1 & $10.1-62.4$ & $<0.0001$ & 9.5 & $2.8-32.4$ & 0.0001 \\
\hline Men having sex with men & 3.9 & $1.4-11.1$ & 0.0963 & 2.0 & $0.5-7.6$ & 0.1017 \\
\hline Other* & 13.2 & $4.4-39.8$ & 0.0062 & 7.0 & $1.5-32.6$ & 0.0898 \\
\hline \multicolumn{7}{|l|}{ Gender } \\
\hline Male & - & - & - & - & - & - \\
\hline Female & 2.3 & $1.6-3.3$ & $<0.0001$ & 0.8 & $0.4-1.5$ & 0.5055 \\
\hline \multicolumn{7}{|l|}{ Year of diagnosis } \\
\hline 1980-1992 & - & - & - & - & - & - \\
\hline $1993-2006$ & 8.3 & $5.0-13.7$ & $<0.0001$ & 4.2 & $2.1-8.3$ & $<0.0001$ \\
\hline \multicolumn{7}{|l|}{ Age } \\
\hline Per 1 year older & 0.9 & $0.8-0.9$ & 0.0003 & 1.0 & $0.9-1.0$ & 0.7923 \\
\hline
\end{tabular}

*Other: transfusion, vertical transmission and unknown risk.

$\mathrm{Cl}$, confidence interval.

as $14.2 \%$ of women (59 of 416) compared with $6.8 \%$ of men (64 of 948) were infected with a non-B variant.

A comparison between subtype B- and non-B-infected patients showed a difference in median age (37vs. 33 years, respectively) $(P<0.0001)$, while $\mathrm{CD} 4$ cell count $(310$ vs. 324 cells $/ \mu \mathrm{L}$, respectively) and plasma viral load (4.04 vs. $4.2 \log$ copies $/ \mathrm{mL}$ ) were comparable in the two groups. The year of diagnosis was significantly associated with the probability of acquiring a non-B clade, as $17.4 \%$ of patients (104 of 597) carried non-B subtypes in the 1993-2006 period compared with $2.5 \%$ (19 of 767) of those in the 1980-1992 period $(P<0.0001)$.

Multivariable analysis confirmed the following independent predictors of higher odds of non-B infection: African ethnicity, heterosexual route of infection and later time of diagnosis (Table 2).

Subtype assignment and clade distribution among ethnicities

A broad heterogeneity of the 417 non-B group M clades was found in patients regardless of their different country of origin. All known pure subtypes, with the exception of $\mathrm{K}$, plus seven distinct CRFs (01, 02, 04, 06, 09, 12 and 13), were detected. The most prevalent pure subtypes were $\mathrm{F}$ [ $n=99$ (23.7\%); $98 \mathrm{~F} 1$ and one F2], A [ $n=53$ (12.7\%); 38 $\mathrm{A} 1$, three A2 and $12 \mathrm{~A} 3], \mathrm{C}(n=47 ; 11.3 \%)$ and $\mathrm{G}(n=23$; 5.5\%). Among CRFs, CRF02_AG and CRF01_AE were the most frequent forms $[n=107(25.7 \%)$ and $n=21$ (5.0\%), respectively]. Thirty-nine URFs (9.3\%), showing complex mosaic patterns, were identified.

The distribution of non-B subtypes differed markedly between patients of European and African origin ( $n=192$ and 146, respectively) (data not shown). The F1 subtype, which was present only in one African individual, was the most frequent clade in Europeans with non-B variants (85 of $192 ; 44.3 \%)$, while the prevalences of A1 $(n=24), \mathrm{C}$ $(n=19)$, CRF02_AG $(n=9)$ and URFs $(n=19)$ were 12.5, 9.9, 4.7 and 9.9\%, respectively. European patients carrying the F1 subtype were mainly Italians $(n=68 ; 82 \%)$ and Romanians ( $n=13 ; 15.7 \%)$.

Among Europeans carrying non-B subtypes, 64.8\% $(n=57)$ were heterosexual and 74.5\% (143 of 192) were male. An association between heterosexual route of infection, but not gender, and non-B clades was found in this group of subjects $(P<0.0001$ and $P=0.46$, respectively).

Differences in the distribution of subtype $\mathrm{B} v s$. individual non-B clades were then analysed for non-B clades detected at a prevalence of $>5 \%$. A significant association with heterosexual route of infection was detected for subtypes F1 and C, with 50\% of F1-infected (17 of 34), 100\% of C-infected (six of six) and 30.6\% of B-infected (528 of 1724) patients being heterosexual $(P=0.006$ for $\mathrm{F} 1 v s$. $\mathrm{B} ; P<0.001$ for $\mathrm{C} v s$. B; $P=0.026$ for $\mathrm{F} 1 v s$. C). No association with gender was detected for any individual clade in Europeans.

Among Africans living in Italy, CRF02_AG was found in $52.1 \%$ of subjects $(n=76)$, followed by C $(n=15 ; 10.3 \%)$, A [10 A3 (6.9\%) and six A1 (4.1\%)], G $(n=13 ; 8.9 \%)$ and B $(n=13 ; 8.2 \%)$ clades and URFs $(n=10 ; 6.9 \%)$. Country 
of origin was known for 102 of these patients. Percentages of immigrants from Ivory Coast, Nigeria, Cameroon and Senegal were 21.6, 21.6, 12.7 and 9.9\%, respectively. The remaining individuals (34.3\%) were from northern $(n=9)$, western $(n=9)$, eastern $(n=10)$, central $(n=5)$ and southern Africa $(n=2)$. Ninety-six (93.2\%) of these patients were heterosexual and the male to female ratio was about 0.5:1 (36:65).

Twenty out of 98 (20.4\%) Latin American patients (52.9\% from Brazil, 15.7\% from Argentina and < 5\% each from Columbia, Ecuador, Peru, Dominican Republic, Cuba, Mexico and Venezuela) harboured non-B strains (11 F1, seven URFs and two C). Nine of 18 subjects from SouthEast Asia (mainly from the Philippines, Thailand and India) harboured non-B subtypes (six CRF01_AE and three C).

\section{Analysis of recombinant HIV-1 variants}

The recombination analysis of 39 URFs identified $13 \mathrm{~B} / \mathrm{F}$, six $G / A$, four $D / B$, three $A / K$, three $G / A / K$, three $C / B$, two CRF02_AG/CRF09_cpx, one CRF02_AG/B, one CRF06_cpx/ CRF02_AG, one CRF18_cpx/B, one $F / C / B$ and one G/CRF09_cpx recombinant.

The proportion of URFs was comparable in Africans (6.8\%), Europeans (9.3\%) and Latin Americans (7.1\%) infected with non-B clades. As expected, URFs were detected in African subjects from Cameroon, Democratic Republic of Congo, Senegal, Nigeria and Ivory Coast.

All B/F recombinants were identified in Italian $(n=8)$ or Brazilian ( $n=5)$ patients.

A complex $\mathrm{G} / \mathrm{U} / \mathrm{F} 1 / \mathrm{B}$ pattern, obtained from a Cuban patient, was found to be a CRF18_cpx/B recombinant, consistent with the patient's country of origin. The CRF06_cpx/CRF02_AG unique recombinant was related to the isolate 00NE-36 from Niger, which has been proposed as the reference sequence for CRF30_cpx (www.hiv.lanl. gov/content/sequence/HIV/CRFs/CRFs.html).

One of two CRF02_AG/CRF09_cpx mosaic forms was harboured by a patient born in Ivory Coast, where this second-generation recombinant has been isolated.

Interestingly, two groups of three sequences each were highly homologous to the MAL (A/K) [23] and the 99GR303 $(\mathrm{G} / \mathrm{A} / \mathrm{K})[24]$ isolates, respectively.

\section{Discussion}

A hallmark of the HIV-1 epidemic in Europe is the substantial increase in non-B clade penetration and circulation that has taken place as a result of the migration flows from subSaharan Africa, South-East Asia and Central and South America that have occurred since the early 1990s [6-13]. In addition to migration, travel to areas with high prevalences of HIV-1 infection, in particular those where commercial sex is widely available, is thought to be responsible for the entry of various group $M$ subtypes into previously subtype B-restricted countries. Italian data from the Centro Operativo AIDS, based on new HIV diagnoses, indicate that the percentage of foreign patients (41.2\% from Africa, 25.2\% from Latin America and 16.1\% from Europe) increased from 11 to $32 \%$ from 1992 to 2007, with heterosexual contact being the most frequent route of infection (increasing from 24.6 to $75.9 \%$ in the same period). Overall, among patients newly diagnosed with HIV-1 infection in the period from 1985 to 2007, the proportion of IDUs declined from 69 to 8.6\%, while sexual transmission increased from 13.3 to $73.7 \%$ and the male to female ratio decreased from 3.5 to 2.5 [18].

The distributions of ethnicity and route of infection in our patient population are in agreement with these data. Moreover, we were able to investigate the relative proportions of heterosexuals and MSM in a large seroprevalence case file mainly covering the central part of Italy. We found that $<3 \%$ of HIV-1-infected patients harboured non-B clades before 1993, as compared with about $20 \%$ in subsequent years. Of note, the probability of acquiring a non-B subtype increased more than 0.25-fold per subsequent year. The prevalence of non-B subtypes in Italy was first estimated in a 2001 study which reported an overall prevalence of 5.4\% among drugnaïve patients, with an increasing trend over time [7]. Two later studies reported higher prevalences of 12.6 and 10.7\% in regions with low/medium and high incidences of infection, respectively $[25,26]$. Both these figures, although showing an increase in non-B prevalence over time, are lower than those reported in this work, as well as in surveillance studies carried out in other European countries such as France, Belgium and the United Kingdom [8,9,11].

According to several studies, the spread of non-B subtypes is highly dependent upon several variables that define the demographics of local HIV-1 epidemics and their evolution over time. The proportions of patients of non-Caucasian ethnicity and those infected via the heterosexual route increased in our case file throughout the study period. However, we also detected a higher prevalence of non-B variants in European individuals after 1992, with a 5-fold increase being found in the proportion of patients with non$B$ variants compared with the earlier period. As expected, the regression analysis indicated a strong association between the African ethnicity and the carriage of non-B strains. However, 50\% of individuals infected with strains other than $\mathrm{B}$ were Caucasian, suggesting that these strains have been onward-transmitted to Europeans at a considerable rate. Overall, an increase in the prevalence of non-B strains was seen in all risk categories; however, the most relevant increase was found in heterosexuals. The multivariable analysis performed on the patient subset with CD showed 
that the heterosexual route of infection was a strong independent predictor of HIV-1 infection with non-B clades, a 9.5-fold higher risk of carriage of non-B infection being found for heterosexuals. Probably because of the local characteristics of the HIV-1 epidemic, such as the high proportion of women among IDUs, the male to female ratio was comparable between the period before 1993 and the period from 1993 onwards (2.25 vs. 2.32, respectively), and female gender was not an independent predictor of non-B infection. Nevertheless, women with non-B variants represented a sizeable proportion (almost one-third) of the total number of women diagnosed after 1992.

Finally, the evaluation of time of HIV-1 diagnosis clearly indicated that the risk of acquiring non-B infection was 4-fold higher for those diagnosed after 1993 as compared with previous years.

High heterogeneity in group $M$ non-B clades was detected in our study, indicating that the sources of nonB infection were dispersed world-wide. Although citizenship was not known for all foreign patients, the detection of specific subtypes in Africans, Latin Americans and SouthEast Asians closely reflected the non-B strains circulating at high prevalence in their countries of origin, suggesting that these subjects were probably infected in their own countries. Notably, our study revealed that the F1 subtype was highly predominant (with a frequency of almost 50\%) among European patients carrying non-B subtypes, more than $80 \%$ of whom were Italians. Specifically, this clade, which has a high prevalence in South America [27] and to some extent in Eastern Europe [28], was found to be significantly associated with the heterosexual route of transmission. This novel finding warrants further investigation, either through collection of information on sexual behaviour or by using phylogenetic approaches to trace the probable origin of these infections. These data will be of value in the development of public health interventions.

An unusually high proportion (about 10\%) of URFs among non-B subtypes were detected in Caucasian and in African and Latin American individuals. This might have been a result of the high accuracy of the phylogenetic and recombination analysis. Indeed, two types of URF were detected in three patients each, making these forms novel CRF candidates to be further characterized by full-length sequencing. URFs with a $\mathrm{B} / \mathrm{F}$ pattern were found at a disproportionately high rate ( $>30 \%$ ), supporting the results of previous studies in which these two clades were found to have a high propensity to recombine $[27,29]$. Further spread of such recombinants may lead to overlapping epidemics, such that the landscape of HIV-1 diversity in Italy may in future be distinct from that of the rest of Europe.

Our methodological approach had some limitations. First, the duration of residence in Italy was not available for immigrants with known countries of origin. Thus, they may have acquired the infection in their country of origin or later in Italy. Similarly, no information about travel was recorded for Italian patients. Secondly, the number of individuals with a known seroconversion date was too small to allow this to be used to determine the date of entry of non-B clades into Italy. Therefore, we used the date of the first HIV-1-positive test as a surrogate for the duration of infection, which is an exceedingly conservative approach; it is probable that entry of non-B clades into Italy and increases in their circulation actually occurred earlier than suggested by our estimates. The increasing proportion of patients presenting late with AIDS further supports this hypothesis, as these patients are diagnosed several years following the acquisition of infection. A third caveat concerns the use of pol sequences for subtype assignment. It is widely accepted that this viral region, encompassing about 1000 nucleotides, is appropriate for use in tracing epidemiological trends in HIV-1-infected patient populations $[19,20]$. Nonetheless, subtyping using the pol gene does not rule out the possibility that other genome regions may belong to different subtypes [30]. The analysis of a limited portion of HIV-1 gene (e.g. pol) confirmed that recombination events occur at high frequency in circulating HIV-1 strains and can influence the distribution of HIV-1 subtypes world-wide [2-4,17].

HIV-1 diversity has an impact on many aspects of HIV infection, including molecular diagnostics [31], cellular tropism [32,33], pathways to drug resistance [34], fitness [35], disease progression [36] and mother-to-child transmission [37]. Despite some limitations, subtype assignment derived from routine drug resistance testing is a fundamental tool for basic surveillance of the spread of HIV-1 clades, with the potential to improve understanding of the biological and clinical features of HIV infection and to enhance prevention strategies.

\section{Acknowledgements}

The authors thank all patients included in the study. This work was supported by grants from the Italian Institute of Health (6th National Programme on HIV/AIDS, contract numbers 40F.56 and 20G.18), from the University of Siena PAR/2005 and from the European Community's FP7 under the project Collaborative HIV and Anti-HIV Drug Resistance Network (CHAIN) (grant agreement 223131).

\section{References}

1 Robertson DL, Sharp PM, McCutchan FE, Hahn BH. Recombination in HIV-1. Nature 1995; 374: 124-126. 
2 Leitner T, Korber B, Daniels M, Calef C, Foley B. HIV-1 Subtype and Circulating Recombinant Form (CRF) Reference Sequences, 2005. Los Alamos National Laboratory, Los Alamos, NM 87545. seq-info@lanl.gov. Available at www.hiv.lanl.gov/content/ sequence/HIV/COMPENDIUM/2005/partI/leitner.pdf (accessed March 2010).

3 Taylor BS, Hammer SM. The challenge of HIV-1 subtype diversity. New Engl J Med 2008; 12: 1590-1602.

4 Hemelaar J, Gouws E, Ghys PD, Osmanov S. Global and regional distribution of HIV-1 genetic subtypes and recombinants in 2004. AIDS 2006; 20: W13-W23.

5 Lukashov VV, Kuiken CL, Vlahov D, Coutinho RA, Goudsmit J. Evidence for HIV type 1 strains of U.S. intravenous drug users as founders of AIDS epidemic among intravenous drug users in northern Europe. AIDS Res Hum Retroviruses 1996; 12: 1179-1183.

6 Sonnerborg A, Durdevic S, Giesecke J, Sällberg M. Dynamics of the HIV-1 subtype distribution in the Swedish HIV-1 epidemic during the period 1980 to 1993. AIDS Res Hum Retroviruses 1997; 13: 343-345.

7 Balotta C, Facchi G, Violin M et al. ICONA study group. Increasing prevalence of non-clade B HIV-1 strains in heterosexual men and women, as monitored by analysis of reverse transcriptase and protease sequences. J Acquir Immune Defic Syndr 2001; 27: 499-505.

8 Chaix ML, Descamps D, Harzic M et al. Stable prevalence of genotypic drug resistance mutations but increase in non-B virus among patients with primary HIV-1 infection in France. AIDS 2003; 17: 2635-2643.

9 Snoeck J, Van Laethem K, Hermans P et al. Rising prevalence of HIV-1 non-B subtypes in Belgium: 1983-2001. J Acquir Immune Defic Syndr 2004; 35: 279-285.

10 Lospitao E, Alvarez A, Soriano V, Holguín A. HIV-1 subtypes in Spain: a retrospective analysis from 1995 to 2003. HIV Med 2005; 6: 313-320.

11 Gifford RJ, de Oliveira T, Rambaut A et al. Phylogenetic surveillance of viral genetic diversity and the evolving molecular epidemiology of human immunodeficiency virus type 1. J Virol 2007; 81: 13050-13056.

12 Palma AC, Araújo F, Duque V, Borges F, Paixão MT, Camacho R. Portuguese SPREAD network. Molecular epidemiology and prevalence of drug resistance-associated mutations in newly diagnosed HIV-1 patients in Portugal. Infect Genet Evol 2007; 7: 391-398.

13 Yerly S, von Wyl V, Ledergerber B et al. Swiss HIV cohort study. Transmission of HIV-1 drug resistance in Switzerland: a 10-year molecular epidemiology survey. AIDS 2007; 21: 2223-2229.

14 Masquelier B, Bhaskaran K, Pillay D et al. CASCADE collaboration. Prevalence of transmitted HIV-1 drug resistance and the role of resistance algorithms: data from seroconverters in the CASCADE collaboration from 1987 to 2003. J Acquir Immune Defic Syndr 2005; 40: 505-511.
15 Wensing AM, Vijver DA, Angarano G et al. Prevalence of drug-resistant HIV-1 variants in untreated individuals in Europe: implications for clinical management. $J$ Infect Dis 2005; 192: 958-966.

16 Wensing AMJ, Vercauteren J, Vijver DA et al. Transmission of drug-resistant HIV-1 in Europe remains limited to single classes. AIDS 2008; 22: 625-635.

17 Thomson MM, Pérez-Alvarez L, Nájera R. Molecular epidemiology of HIV-1 genetic forms and its significance for vaccine development and therapy. Lancet Infect Dis 2002; 2: 461-471.

18 Notiziario COA Marzo 2009. Available at www.iss.it/binary/ publ/cont/COAonline.pdf (accessed March 2010).

19 Lemey P, Derdelinckx I, Rambaut A et al. Molecular footprint of drug-selective pressure in a human immunodeficiency virus transmission chain. J Virol 2005; 79: 11981-11989.

20 Hué S, Gifford RJ, Dunn D, Fernhill E, Pillay D. UK collaborative group on HIV drug resistance. Demonstration of sustained drug-resistant human immunodeficiency virus type 1 lineages circulating among treatment-naïve individuals. J Virol 2009; 83: 2645-2654.

21 Zazzi M, Riccio ML, Venturi G et al. Long-read direct infrared sequencing of crude PCR products for prediction of resistance to HIV-1 reverse transcriptase and protease inhibitors. $\mathrm{Mol}$ Biotechnol 1998; 10: 1-8.

22 Peduzzi C, Pierotti P, Venturi G et al. Performance of an in-house genotypic antiretroviral resistance assay in patients pretreated with multiple human immunodeficiency virus type 1 protease and reverse transcriptase inhibitors. J Clin Virol 2002; 25: 57-62.

23 Oelrichs RB, Workman C, Laukkanen T, McCutchan FE, Deacon NJ. A novel subtype A/G/J recombinant full-length HIV type 1 genome from Burkina Faso. AIDS Res Hum Retroviruses 1998; 14: 1495-1500.

24 Nasioulas G, Paraskevis D, Magiorkinis E, Theodoridou M, Hatzakis A. Molecular analysis of the full-length genome of HIV type 1 subtype I: evidence of A/G/I recombination. AIDS Res Hum Retroviruses 1999; 15: 745-758.

25 Monno L, Brindicci G, Lo Caputo S et al. HIV-1 subtypes and circulating recombinant forms (CRFs) from HIV-infected patients residing in two regions of central and southern Italy. J Med Virol 2005; 75: 483-490.

26 Baldanti F, Paolucci S, Ravasi G et al. Changes in circulation of $B$ and non-B HIV strains: spotlight on a reference centre for infectious diseases in Northern Italy. J Med Virol 2008; 80: 947-952.

27 Thomson MM, Nájera R. Molecular epidemiology of HIV-1 variants in the global AIDS pandemic: an update. AIDS Rev 2005; 7: 210-224.

28 Op De Coul E, van den Burg R, Asjö B et al. Genetic evidence of multiple transmissions of HIV type 1 subtype F within Romania from adult blood donors to children. AIDS Res Hum Retroviruses 2000; 16: 327-336. 
29 Thomson M, Sierra M, Tanuri A et al. Analysis of near fulllength genome sequences of HIV type $1 \mathrm{BF}$ intersubtype recombinant viruses from Brazil reveals their independent origins and their lack of relationship to CRF12_BF. AIDS Res Hum Retroviruses 2004; 20: 1126-1133.

30 Razzolini F, Vicenti I, Saladini F et al. Natural variability in the HR-1 and HR-2 domains of HIV type 1 gp41 from different clades circulating in Italy. AIDS Res Hum Retroviruses 2007; 23: 558-563.

31 Holguín A, López M, Molinero M, Soriano V. Performance of three commercial viral load assays, versant human immunodeficiency virus type 1 (HIV-1) RNA bDNA v3.0, Cobas AmpliPrep/Cobas TaqMan HIV-1, and NucliSens HIV-1 EasyQ v1.2, testing HIV-1 non-B subtypes and recombinant variants. J Clin Microbiol 2008; 46: 2918-2923.

32 Lobritz MA, Marozsan AJ, Troyer RM, Arts EJ. Natural variation in the $\mathrm{V} 3$ crown of human immunodeficiency virus type 1 affects replicative fitness and entry inhibitor sensitivity. J Virol 2007; 81: 8258-8269.

33 Huang W, Eshleman SH, Toma J et al. Coreceptor tropism in human immunodeficiency virus type 1 subtype D: high prevalence of CXCR4 tropism and heterogeneous composition of viral populations. J Virol 2007; 81:

7885-7893.

34 Martinez-Cajas JL, Pai NP, Klein MB, Wainberg MA. Differences in resistance mutations among HIV-1 non-subtype B infections: a systematic review of evidence (1996-2008).

J Int AIDS Soc 2009; 12: 1-11.

35 Ariën KK, Abraha A, Quiñones-Mateu ME, Kestens L, Vanham G, Arts EJ. The replicative fitness of primary human immunodeficiency virus type 1 (HIV-1) group M, HIV-1 group 0, and HIV-2 isolates. $J$ Virol 2005; 79: 8979-8990.

36 Keller M, Lu Y, Lalonde RG, Klein MB. Impact of HIV-1 viral subtype on CD4 + T-cell decline and clinical outcomes in antiretroviral naive patients receiving universal healthcare. AIDS 2009; 23: 731-737.

37 Eshleman SH, Guay LA, Mwatha A et al. Comparison of mother-to-child transmission rates in Ugandan women with subtype A versus D HIV-1 who received single-dose nevirapine prophylaxis: HIV network for prevention trials 012. J Acquir Immune Defic Syndr 2005; 39: 593-597. 